\title{
INDESTRUCTIBILITY AND THE LEVINSKI PROPERTY
}

\author{
ARTHUR W. APTER
}

\begin{abstract}
We investigate some possible interactions between an indestructibly supercompact cardinal and a generalization of a property originally due to Levinski [18].
\end{abstract}

\section{INTRODUCTION AND PRELIMINARIES}

We begin with some terminology and notational conventions. Suppose $\kappa$ is a regular cardinal. For $\beta$ an arbitrary ordinal, the partial ordering $\operatorname{Add}(\kappa, \beta)$ is the standard Cohen partial ordering for adding $\beta$ many Cohen subsets of $\kappa$. The partial ordering $\mathbb{P}$ is $\kappa$-directed closed if for every directed set $D \subseteq \mathbb{P}$ of size less than $\kappa$, there is a condition in $\mathbb{P}$ extending each member of $D$. Say that a measurable cardinal $\kappa$ satisfies the Levinski property $L P$ if $2^{\kappa}=\kappa^{+}$, yet GCH fails on some final segment of inaccessible cardinals below $\kappa$. Call an ordinal $\alpha>0 \operatorname{good}$ if $\alpha$ is definable and is also such that for any cardinal $\delta, \delta^{+\alpha}$ is a regular cardinal below the least inaccessible cardinal above $\delta .^{1}$ Refine the preceding by saying that a measurable cardinal $\kappa$ satisfies the Levinski property $L P(\alpha)$ for a fixed but arbitrary good ordinal $\alpha$ if for every inaccessible cardinal $\delta$ in some final segment below $\kappa, 2^{\delta}=\delta^{+\alpha}$, yet $2^{\kappa}=\kappa^{+}$.

2010 Mathematics Subject Classification. 03E35, 03E55.

Key words and phrases. Supercompact cardinal, indestructibility, lottery sum, Levinski property.

The author's research was partially supported by PSC-CUNY grants.

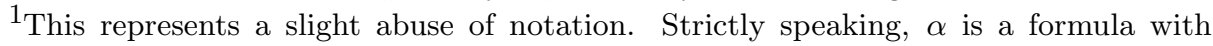
parameter $\delta$, which we will suppress. Examples of good ordinals include 2, 3, 75, $\omega+1$, etc. This is since 2, 3, 75, and $\omega+1$ are all definable. Further, for $\alpha$ any of these values, $\delta^{+\alpha}$ is regular since it is a successor cardinal. $\delta^{+\alpha}$ is also below the least inaccessible cardinal above $\delta$, which must be a (regular) limit cardinal.

Copyright (c) 2016 by ANUBIH. 
Both LP and $\operatorname{LP}(\alpha)$ are variants of a property first studied by Levinski in [18].

Beginning now our main narrative, it is an interesting and curious fact that the large cardinal structure of the universe above a supercompact cardinal $\kappa$ with suitable indestructibility properties can affect the large cardinal structure below $\kappa$ in quite surprising ways. On the other hand, these effects may be mitigated if the universe contains relatively few large cardinals. These sorts of occurrences have previously been investigated in $[2,3,4,5,6,7,1,8]$.

The purpose of this paper is to continue studying this phenomenon, but in the context of different versions of the Levinski property together with their interactions with an indestructibly supercompact cardinal. We begin with the following theorem, where as in [17], $\kappa$ is indestructibly supercompact if $\kappa$ 's supercompactness is preserved by arbitrary $\kappa$-directed closed forcing.

Theorem 1. Suppose that $\kappa$ is indestructibly supercompact and there is a measurable cardinal $\lambda>\kappa$. Then for any good ordinal $\alpha, A_{\alpha}=\{\delta<\kappa \mid \delta$ is measurable, $\delta$ is not a limit of measurable cardinals, and $L P(\alpha)$ holds for $\delta\}$ is unbounded in $\kappa$.

The large cardinal hypothesis on $\lambda$ is necessary, as we further demonstrate by constructing via forcing models containing an indestructibly supercompact cardinal $\kappa$ with no measurable cardinal above it in which for fixed but arbitrary good $\alpha$, every measurable cardinal $\delta<\kappa$ which is not a limit of measurable cardinals satisfies $\operatorname{LP}(\alpha)$. Specifically we have:

Theorem 2. Suppose $V \vDash " Z F C+\kappa$ is supercompact + No cardinal $\zeta>\kappa$ is measurable". Let $\alpha$ be a good ordinal. There is then a partial ordering $\mathbb{P} \in V$ such that $V^{\mathbb{P}} \vDash " Z F C+$ No cardinal $\zeta>\kappa$ is measurable $+\kappa$ is indestructibly supercompact + If $\delta<\kappa$ is a measurable cardinal which is not a limit of measurable cardinals, then $L P(\alpha)$ holds".

We also show the necessity of the large cardinal hypothesis on $\lambda$ by constructing via forcing models containing an indestructibly supercompact cardinal $\kappa$ with no measurable cardinals above it such that for every measurable 
cardinal $\delta<\kappa$ which is not a limit of measurable cardinals, $2^{\delta}>\delta^{+}$. In particular we have:

Theorem 3. Suppose $V \vDash " Z F C+\kappa$ is supercompact + No cardinal $\zeta>\kappa$ is measurable". There is then a partial ordering $\mathbb{P} \subseteq V$ such that $V^{\mathbb{P}} \vDash$ "ZFC + No cardinal $\zeta>\kappa$ is measurable $+\kappa$ is indestructibly supercompact + If $\delta<\kappa$ is a measurable cardinal which is not a limit of measurable cardinals, then $2^{\delta}=\delta^{++"}$.

We take this opportunity to make a few remarks concerning Theorems 2 and 3. In the conclusions of Theorem 3, there is nothing special about having $2^{\delta}=\delta^{++}$for every measurable cardinal $\delta<\kappa$ which is not a limit of measurable cardinals. As our methods of proof will show, it is also possible to have $2^{\delta}=\delta^{+3}, 2^{\delta}=\delta^{+4}$, etc. In addition, for both Theorems 2 and 3 , the measurable cardinal $\delta<\kappa$ cannot in general be a limit of measurable cardinals. This is since for any $n<\omega,\left(\operatorname{Add}\left(\kappa^{+}, 1\right) * \operatorname{Add}\left(\kappa, \kappa^{+n}\right)\right)^{V^{\mathbb{P}}}$ is $\kappa$-directed closed in $V^{\mathbb{P}}$. Standard arguments (see [14, Exercise 15.16]) show that after forcing with $\operatorname{Add}\left(\kappa^{+}, 1\right), 2^{\kappa}=\kappa^{+}$. Thus, if $V^{\mathbb{P}} \vDash " \kappa$ is

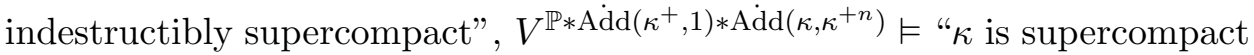
$+2^{\kappa}=\kappa^{+n}+\kappa$ is a measurable limit of measurable cardinals". Hence, by reflection, in $V^{\mathbb{P} * \operatorname{Add}\left(\kappa^{+}, 1\right) * \operatorname{Add}\left(\kappa, \kappa^{+n}\right)}, B_{n}=\{\delta<\kappa \mid \delta$ is a measurable limit of measurable cardinals and $\left.2^{\delta}=\delta^{+n}\right\}$ is unbounded in $\kappa$. Because $\left(\operatorname{Add}\left(\kappa^{+}, 1\right) * \operatorname{Add}\left(\kappa, \kappa^{+n}\right)\right)^{V^{\mathbb{P}}}$ is $\kappa$-directed closed in $V^{\mathbb{P}}, B_{n}$ is unbounded in $V^{\mathbb{P}}$ as well. This precludes the conclusions of both Theorems 2 and 3 holding for $\delta$ when $\delta<\kappa$ is a measurable limit of measurable cardinals.

We conclude Section 1 with a very brief discussion of some preliminary material. We presume a basic knowledge of large cardinals and forcing. A good reference in this regard is [14]. When forcing, $q \geq p$ means that $q$ is stronger than $p$. We will have two slight abuses of notation. In particular, when $G$ is $V$-generic over $\mathbb{P}$, we take both $V[G]$ and $V^{\mathbb{P}}$ as being the generic extension of $V$ by $\mathbb{P}$. We also occasionally confuse terms with the sets they denote, especially for ground model sets and variants of the generic object. For $\alpha<\beta$ ordinals, $[\alpha, \beta)$ and $(\alpha, \beta)$ are as in standard interval notation.

We recall for the benefit of readers the definition given by Hamkins in $[13$, Section 3] of the lottery sum of a collection of partial orderings. If $\mathfrak{A}$ is 
a collection of partial orderings, then the lottery sum is the partial ordering $\oplus \mathfrak{A}=\{\langle\mathbb{P}, p\rangle \mid \mathbb{P} \in \mathfrak{A}$ and $p \in \mathbb{P}\} \cup\{0\}$, ordered with 0 below everything and $\langle\mathbb{P}, p\rangle \leq\left\langle\mathbb{P}^{\prime}, p^{\prime}\right\rangle$ iff $\mathbb{P}=\mathbb{P}^{\prime}$ and $p \leq p^{\prime}$. Intuitively, if $G$ is $V$-generic over $\oplus \mathfrak{A}$, then $G$ first selects an element of $\mathfrak{A}$ (or as Hamkins says in [13], "holds a lottery among the posets in $\mathfrak{A}$ ") and then forces with it. ${ }^{2}$

A corollary of Hamkins' work on gap forcing found in [11, 12] will be employed in the proof of Theorems 2 and 3. We therefore state as a separate theorem what is relevant for this paper, along with some associated terminology, quoting from $[11,12]$ when appropriate. Suppose $\mathbb{P}$ is a partial ordering which can be written as $\mathbb{Q} * \dot{\mathbb{R}}$, where $|\mathbb{Q}|<\delta, \mathbb{Q}$ is nontrivial, and $\vdash_{\mathbb{Q}}$ " $\mathbb{R}$ is $\delta^{+}$-directed closed". In Hamkins' terminology of $[11,12], \mathbb{P}$ admits a gap at $\delta$. Also, as in the terminology of $[11,12]$ and elsewhere, an embedding $j: \bar{V} \rightarrow \bar{M}$ is amenable to $\bar{V}$ when $j\lceil A \in \bar{V}$ for any $A \in \bar{V}$. The specific corollary of Hamkins' work from [11, 12] we will be using is then the following.

Theorem 4. (Hamkins) Suppose that $V[G]$ is a generic extension obtained by forcing with $\mathbb{P}$ that admits a gap at some regular $\delta<\kappa$. Suppose further that $j: V[G] \rightarrow M[j(G)]$ is an elementary embedding with critical point $\kappa$ for which $M[j(G)] \subseteq V[G]$ and $M[j(G)]^{\delta} \subseteq M[j(G)]$ in $V[G]$. Then $M \subseteq V$; indeed, $M=V \cap M[j(G)]$. If the full embedding $j$ is amenable to $V[G]$, then the restricted embedding $j \uparrow V: V \rightarrow M$ is amenable to $V$. If $j$ is definable from parameters (such as a measure or extender) in $V[G]$, then the restricted embedding $j \uparrow V$ is definable from the names of those parameters in $V$.

A consequence of Theorem 4 is that if $\mathbb{P}$ admits a gap at some regular $\delta<\kappa$ and $V^{\mathbb{P}} \vDash$ " $\kappa$ is measurable", then $V \vDash$ " $\kappa$ is measurable" as well.

\section{The proofs of theorems $1-3$}

We turn now to the proof of Theorem 1, whose proof will depend on the existence of a certain partial ordering $\mathbb{P}(\delta, \lambda, \alpha)$. We isolate the existence of this key forcing notion in the following theorem.

\footnotetext{
${ }^{2}$ The terminology "lottery sum" is due to Hamkins, although the concept of the lottery sum of partial orderings has been around for quite some time and has been referred to at different junctures via the names "disjoint sum of partial orderings," "side-by-side forcing," and "choosing which partial ordering to force with generically."
} 
Theorem 5. Suppose $V \vDash " \delta<\lambda$ are such that $\delta$ is a regular cardinal and $\lambda$ is the least measurable cardinal greater than $\lambda$ ". Let $\alpha$ be a good ordinal. There is then a $\delta$-directed closed partial ordering $\mathbb{P}(\delta, \lambda, \alpha)$ such that $V^{\mathbb{P}(\delta, \lambda, \alpha) \vDash " \lambda}$ is the least measurable cardinal greater than $\delta+L P(\alpha)$ holds for $\lambda "$.

Proof. Assume $\delta, \lambda$, and $\alpha$ are as in the hypotheses of Theorem 5. We define $\mathbb{P}(\delta, \lambda, \alpha)$ as $\mathbb{P}^{1} * \dot{\mathbb{P}}^{2}$, where $\mathbb{P}^{1}=\operatorname{Add}\left(\lambda^{+}, 1\right)$. Because $\operatorname{Add}\left(\lambda^{+}, 1\right)$ is $\lambda^{+}$-directed closed, $V^{\mathbb{P}^{1}} \vDash$ " $\lambda$ is the least measurable cardinal greater than $\delta$ ". As we have already observed, standard arguments show that $V^{\mathbb{P}^{1}} \vDash$ " $2^{\lambda}=\lambda^{+} "$.

Work now in $\bar{V}=V^{\mathbb{P}^{1}}$. $\mathbb{P}^{2}$ is defined as $\mathbb{P}_{\lambda} * \operatorname{Add}\left(\lambda, \lambda^{+}\right)$, where $\mathbb{P}_{\lambda}=$ $\left\langle\left\langle\mathbb{P}_{\beta}, \dot{\mathbb{Q}}_{\beta}\right\rangle \mid \beta<\lambda\right\rangle$ is the reverse Easton iteration of length $\lambda$ which begins by forcing with $\operatorname{Add}(\delta, 1)$ and then does nontrivial forcing only at those $\gamma \in(\delta, \lambda)$ which are inaccessible cardinals in $\bar{V}$. At such a stage $\gamma, \dot{\mathbb{Q}}_{\gamma}$ is a term for $\operatorname{Add}\left(\gamma^{+}, 1\right) * \operatorname{Add}\left(\gamma, \gamma^{+\alpha}\right)$. Standard arguments once again show that $\bar{V}^{\mathbb{P}^{1}} \vDash " 2^{\gamma}=\gamma^{+\alpha}$ if $\gamma \in(\delta, \lambda)$ is inaccessible". In addition, by its definition, $\mathbb{P}(\delta, \lambda, \alpha)$ is $\delta$-directed closed.

It is also the case that $\bar{V}^{\mathbb{P}^{2}} \vDash$ " $\lambda$ is measurable". To see this, let $j$ : $\bar{V} \rightarrow M$ be an elementary embedding witnessing the measurability of $\lambda$ in $\bar{V}$ generated by a normal measure over $\lambda$. In particular, $M^{\lambda} \subseteq M$. We combine several ideas (including a standard lifting argument, an idea due to Levinski [18], and an idea due to Magidor [20]) to show that $j$ lifts in $\bar{V}^{\mathbb{P}_{\lambda} * \operatorname{Add}\left(\lambda, \lambda^{+}\right)}$ to $j: \bar{V}^{\mathbb{P}_{\lambda} * \operatorname{Add}\left(\lambda, \lambda^{+}\right)} \rightarrow M^{j\left(\mathbb{P}_{\lambda} * \operatorname{Add}\left(\lambda, \lambda^{+}\right)\right)}$. Specifically, let $G_{0}$ be $\bar{V}$-generic over $\mathbb{P}_{\lambda}$, and let $G_{1}$ be $\bar{V}\left[G_{0}\right]$-generic over $\operatorname{Add}\left(\lambda, \lambda^{+}\right)$. Observe that $j\left(\mathbb{P}_{\lambda} *\right.$ $\left.\operatorname{Add}\left(\lambda, \lambda^{+}\right)\right)=\mathbb{P}_{\lambda} * \operatorname{Add}\left(\lambda^{+}, 1\right) * \operatorname{Add}\left(\lambda, \lambda^{+\alpha}\right) * \dot{\mathbb{Q}} * \operatorname{Add}\left(j(\lambda), j\left(\lambda^{+}\right)\right)$. Working in $\bar{V}\left[G_{0}\right]$, we first note that since $\mathbb{P}_{\lambda}$ is $\lambda$-c.c., $M\left[G_{0}\right]$ remains $\lambda$-closed with respect to $\bar{V}\left[G_{0}\right]$. This means that $\left(\operatorname{Add}\left(\lambda^{+}, 1\right)\right)^{M\left[G_{0}\right]}$ (which by the $\lambda$ closure of $M\left[G_{0}\right]$ with respect to $\bar{V}\left[G_{0}\right]$ has the same definition in both $\bar{V}\left[G_{0}\right]$ and $\left.M\left[G_{0}\right]\right)$ is $\lambda^{+}$-directed closed in both $M\left[G_{0}\right]$ and $\bar{V}\left[G_{0}\right]$. Consequently, without fear of ambiguity, we write $\operatorname{Add}\left(\lambda^{+}, 1\right)$ for $\left(\operatorname{Add}\left(\lambda^{+}, 1\right)\right)^{M\left[G_{0}\right]}$.

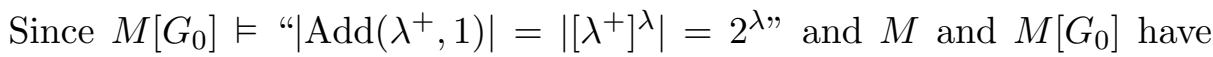
the same cardinals at and above $\lambda$, the number of dense open subsets of $\operatorname{Add}\left(\lambda^{+}, 1\right)$ present in $M\left[G_{0}\right]$ is $\left(2^{2^{\lambda}}\right)^{M}<j(\lambda)$. In $\bar{V}$, since $M$ is given 
via an ultrapower by a normal measure over $\lambda,|j(\lambda)|$ may be calculated as $|\{f \mid f: \lambda \rightarrow \lambda\}|=2^{\lambda}=\lambda^{+}$. Since $\lambda^{+}$is preserved from $\bar{V}$ to $\bar{V}\left[G_{0}\right]$, we may let $\left\langle D_{\beta}\right| \beta\left\langle\lambda^{+}\right\rangle \in \bar{V}\left[G_{0}\right]$ enumerate the dense open subsets of $\operatorname{Add}\left(\lambda^{+}, 1\right)$ present in $M\left[G_{0}\right]$. We may now use the fact that $\operatorname{Add}\left(\lambda^{+}, 1\right)$ is $\lambda^{+}$-directed closed in $\bar{V}\left[G_{0}\right]$ to meet each $D_{\beta}$ and thereby construct in $\bar{V}\left[G_{0}\right]$ an $M\left[G_{0}\right]$-generic object $H_{0}$ over $\operatorname{Add}\left(\lambda^{+}, 1\right)$. Our construction guarantees that $j^{\prime \prime} G_{0} \subseteq G_{0} * H_{0}$, so $j$ lifts in $\bar{V}\left[G_{0}\right]$ to $j: \bar{V}\left[G_{0}\right] \rightarrow M\left[G_{0}\right]\left[H_{0}\right]$. Note that because $\operatorname{Add}\left(\lambda^{+}, 1\right)$ is $\lambda^{+}$-directed closed in both $M\left[G_{0}\right]$ and $\bar{V}\left[G_{0}\right]$, $M\left[G_{0}\right]\left[H_{0}\right]$ remains $\lambda$-closed with respect to $\bar{V}\left[G_{0}\right]\left[H_{0}\right]=\bar{V}\left[G_{0}\right]$.

We use now Levinski's ideas of [18] to show that it is possible to rearrange $G_{1}$ to form an $M\left[G_{0}\right]\left[H_{0}\right]$-generic object $H_{1}$ over $\left(\operatorname{Add}\left(\lambda, \lambda^{+\alpha}\right)\right)^{M\left[G_{0}\right]\left[H_{0}\right]}$ in $\bar{V}\left[G_{0}\right]\left[G_{1}\right]$. Since $\bar{V} \vDash " 2^{\lambda}=\lambda^{+}$" and $j$ is generated by an ultrafilter over $\lambda, \lambda^{+}<j(\lambda)<\lambda^{++}$. In particular, because $M\left[G_{0}\right] \vDash " \lambda^{+} \leq$ $2^{\lambda}=\left|\left(\operatorname{Add}\left(\lambda^{+}, 1\right)\right)\right|<j(\lambda)$ ", any $\gamma \in\left(\lambda^{+}, j(\lambda)\right)$ which $M\left[G_{0}\right]\left[H_{0}\right]$ believes to be a cardinal actually is an ordinal of cardinality $\lambda^{+}$in either $\bar{V}, \bar{V}\left[G_{0}\right]$, or $\bar{V}\left[G_{0}\right]\left[G_{1}\right]$. Hence, $\bar{V}\left[G_{0}\right]\left[G_{1}\right] \vDash "\left|\left(\lambda^{+\alpha}\right)^{M\left[G_{0}\right]\left[H_{0}\right]}\right|=\lambda^{+}$". Let $\left(\lambda^{+\alpha}\right)^{M\left[G_{0}\right]\left[H_{0}\right]}=\rho$. Working in $\bar{V}\left[G_{0}\right]\left[G_{1}\right]$, we may therefore let $f: \lambda^{+} \rightarrow \rho$ be a bijection. For any $p \in \operatorname{Add}\left(\lambda, \lambda^{+}\right), g(p)=\{\langle\langle\sigma, f(\beta)\rangle, \gamma\rangle \mid\langle\langle\sigma, \beta\rangle, \gamma\rangle \in$ $p\} \in(\operatorname{Add}(\lambda, \rho))^{M\left[G_{0}\right]\left[H_{0}\right]}$. As can be easily checked (see [18]), $G_{1}=\{g(p) \mid$ $p \in H\}$ is an $M\left[G_{0}\right]\left[H_{0}\right]$-generic object over $(\operatorname{Add}(\lambda, \rho))^{M\left[G_{0}\right]\left[H_{0}\right]}$. As before, our construction guarantees that $j^{\prime \prime} G_{0} \subseteq G_{0} * H_{0} * H_{1}$, so $j$ lifts in $\bar{V}\left[G_{0}\right]\left[G_{1}\right]$ to $j: \bar{V}\left[G_{0}\right] \rightarrow M\left[G_{0}\right]\left[H_{0}\right]\left[H_{1}\right]$.

Because $\left(\operatorname{Add}\left(\lambda, \lambda^{+}\right)\right)^{\bar{V}\left[G_{0}\right]}$ is $\lambda^{+}$-c.c. in $\bar{V}\left[G_{0}\right], M\left[G_{0}\right]\left[H_{0}\right]\left[H_{1}\right]$ remains $\lambda$-closed with respect to $\bar{V}\left[G_{0}\right]\left[G_{1}\right]$. In addition, since $M\left[G_{0}\right]\left[H_{0}\right]\left[H_{1}\right] \vDash$ "Q⿱Q is a reverse Easton iteration of length $j(\lambda) ", M\left[G_{0}\right]\left[H_{0}\right]\left[H_{1}\right] \vDash "|\mathbb{Q}|=j(\lambda)$ and $\mathbb{Q}$ is $j(\lambda)$-c.c.". This means the number of antichains of $\mathbb{Q}$ present in $M\left[G_{0}\right]\left[H_{0}\right]\left[H_{1}\right]$ is $j(\lambda)$. Further, as $M\left[G_{0}\right]\left[H_{0}\right]\left[H_{1}\right] \vDash " \mathbb{Q}$ is $\lambda^{+}$-directed closed" and $M\left[G_{0}\right]\left[H_{0}\right]\left[H_{1}\right]$ is $\lambda$-closed with respect to $\bar{V}\left[G_{0}\right]\left[G_{1}\right], \mathbb{Q}$ is $\lambda^{+}$-directed closed in $\bar{V}\left[G_{0}\right]\left[G_{1}\right]$ as well. This means that the argument used in the construction of $H_{0}$ may be used to construct in $\bar{V}\left[G_{0}\right]\left[G_{1}\right]$ an $M\left[G_{0}\right]\left[H_{0}\right]\left[H_{1}\right]$-generic object $H_{2}$ over $\mathbb{Q}$. Since once again it is the case that $j^{\prime \prime} G_{0} \subseteq G_{0} * H_{0} * H_{1} * H_{2}$, we may then in $\bar{V}\left[G_{0}\right]\left[G_{1}\right]$ lift $j$ to $j: \bar{V}\left[G_{0}\right] \rightarrow M\left[G_{0}\right]\left[H_{0}\right]\left[H_{1}\right]\left[H_{2}\right]$. By the fact that $\mathbb{Q}$ is $\lambda^{+}$-directed closed 
in both $M\left[G_{0}\right]\left[H_{0}\right]\left[H_{1}\right]$ and $\bar{V}\left[G_{0}\right]\left[G_{1}\right], M\left[G_{0}\right]\left[H_{0}\right]\left[H_{1}\right]\left[H_{2}\right]$ remains $\lambda$-closed with respect to $\bar{V}\left[G_{0}\right]\left[G_{1}\right]\left[H_{2}\right]=\bar{V}\left[G_{0}\right]\left[H_{0}\right]\left[G_{1}\right]\left[H_{2}\right]=\bar{V}\left[G_{0}\right]\left[G_{1}\right]$.

We now use arguments originally due to Magidor [20], which are also given in [9, pages 119-120] and are found other places in the literature as well, to construct in $\bar{V}\left[G_{0}\right]\left[G_{1}\right]$ an $M\left[G_{0}\right]\left[H_{0}\right]\left[H_{1}\right]\left[H_{2}\right]$-generic object $H_{3}$ over $\left(\operatorname{Add}\left(j(\lambda), j\left(\lambda^{+}\right)\right)^{M\left[G_{0}\right]\left[H_{0}\right]\left[H_{1}\right]\left[H_{2}\right]}\right.$. For the convenience of readers, we present these arguments below.

For $\zeta \in\left(\lambda, \lambda^{+}\right)$and $p \in \operatorname{Add}\left(\lambda, \lambda^{+}\right)$, let $p\lceil\zeta=\{\langle\langle\rho, \sigma\rangle, \eta\rangle \in p \mid \sigma<\zeta\}$ and $G_{1}\left\lceil\zeta=\left\{p\left\lceil\zeta \mid p \in G_{1}\right\}\right.\right.$. Clearly, $\bar{V}\left[G_{0}\right]\left[G_{1}\right] \vDash " \mid G_{1}\lceil\zeta \mid \leq \lambda$ for all $\zeta \in\left(\lambda, \lambda^{+}\right)$". Thus, since $\operatorname{Add}\left(j(\lambda), j\left(\lambda^{+}\right)\right)^{M\left[G_{0}\right]\left[H_{0}\right]\left[H_{1}\right]\left[H_{2}\right]}$ is $j(\lambda)$ directed closed and $j(\lambda)>\lambda^{+}, q_{\zeta}=\bigcup\left\{j(p) \mid p \in G_{1}\lceil\zeta\}\right.$ is well-defined and is an element of $\operatorname{Add}\left(j(\lambda), j\left(\lambda^{+}\right)\right)^{M\left[G_{0}\right]\left[H_{0}\right]\left[H_{1}\right]\left[H_{2}\right]}$. Further, if $\langle\rho, \sigma\rangle \in$ $\operatorname{dom}\left(q_{\zeta}\right)-\operatorname{dom}\left(\bigcup_{\beta<\zeta} q_{\beta}\right) \quad\left(\bigcup_{\beta<\zeta} q_{\beta}\right.$ is well-defined by closure), then $\sigma \in$ $\left[\bigcup_{\beta<\zeta} j(\beta), j(\zeta)\right)$. To see this, assume to the contrary that $\sigma<\bigcup_{\beta<\zeta} j(\beta)$. Let $\beta$ be minimal such that $\sigma<j(\beta)$. It must thus be the case that for some $p \in G_{1}\lceil\zeta,\langle\rho, \sigma\rangle \in \operatorname{dom}(j(p))$. Since by elementarity and the definitions of $G_{1}\left\lceil\beta\right.$ and $G_{1}\left\lceil\zeta\right.$, for $p\left\lceil\beta=q \in G_{1} \uparrow \beta, j(q)=j(p) \uparrow j(\beta)=j(p \uparrow \beta)\right.$, it must be the case that $\langle\rho, \sigma\rangle \in \operatorname{dom}(j(q))$. This means $\langle\rho, \sigma\rangle \in \operatorname{dom}\left(q_{\beta}\right)$, a contradiction.

Since $M\left[G_{0}\right]\left[H_{0}\right]\left[H_{1}\right]\left[H_{2}\right] \vDash " j(\lambda)$ is inaccessible and $2^{j(\lambda)}=j\left(\lambda^{+}\right)$", an application of $[14$, Lemma 15.4$]$ shows that $M\left[G_{0}\right]\left[H_{0}\right]\left[H_{1}\right]\left[H_{2}\right] \vDash " A d d(j(\lambda)$, $\left.j\left(\lambda^{+}\right)\right)$is $j\left(\lambda^{+}\right)$-c.c. and has $j\left(\lambda^{+}\right)$many maximal antichains". This means that if $\mathcal{A} \in M\left[G_{0}\right]\left[H_{0}\right]\left[H_{1}\right]\left[H_{2}\right]$ is a maximal antichain of $\operatorname{Add}\left(j(\lambda), j\left(\lambda^{+}\right)\right)$, $\mathcal{A} \subseteq \operatorname{Add}(j(\lambda), \beta)$ for some $\beta \in\left(j(\lambda), j\left(\lambda^{+}\right)\right)$. Thus, since the fact $\bar{V} \vDash$ " $2^{\lambda}=\lambda^{+}$" and the fact $j$ is generated by a normal measure over $\lambda$ imply that $V \vDash "\left|j\left(\lambda^{+}\right)\right|=\lambda^{+}$", we can let $\left\langle\mathcal{A}_{\zeta} \mid \zeta \in\left(\lambda, \lambda^{+}\right)\right\rangle \in \bar{V}\left[G_{0}\right]\left[G_{1}\right]$ be an enumeration of all of the maximal antichains of $\operatorname{Add}\left(j(\lambda), j\left(\lambda^{+}\right)\right)$present in $M\left[G_{0}\right]\left[H_{0}\right]\left[H_{1}\right]\left[H_{2}\right]$.

Working in $\bar{V}\left[G_{0}\right]\left[G_{1}\right]$, we define now an increasing sequence $\left\langle r_{\zeta}\right| \zeta \in$ $\left.\left(\lambda, \lambda^{+}\right)\right\rangle$of elements of $\operatorname{Add}\left(j(\lambda), j\left(\lambda^{+}\right)\right)$such that $\forall \zeta \in\left(\lambda, \lambda^{+}\right)\left[r_{\zeta} \geq q_{\zeta}\right.$ and $\left.r_{\zeta} \in \operatorname{Add}(j(\lambda), j(\zeta))\right]$ and such that $\forall \mathcal{A} \in\left\langle\mathcal{A}_{\zeta} \mid \zeta \in\left(\lambda, \lambda^{+}\right)\right\rangle \exists \beta \in$ $\left(\lambda, \lambda^{+}\right) \exists r \in \mathcal{A}\left[r_{\beta} \geq r\right]$. Assuming we have such a sequence, $H_{3}=\{p \in$ $\operatorname{Add}\left(j(\lambda), j\left(\lambda^{+}\right)\right) \mid \exists r \in\left\langle r_{\zeta} \mid \zeta \in\left(\lambda, \lambda^{+}\right)\right\rangle[r \geq p]$ is an $M\left[G_{0}\right]\left[H_{0}\right]\left[H_{1}\right]\left[H_{2}\right]-$ generic object over $\operatorname{Add}\left(j(\lambda), j\left(\lambda^{+}\right)\right)$. To define $\left\langle r_{\zeta} \mid \zeta \in\left(\lambda, \lambda^{+}\right)\right\rangle$, if $\zeta$ is a 
limit, we let $r_{\zeta}=\bigcup_{\beta \in(\lambda, \zeta)} r_{\beta}$. By the facts $\left\langle r_{\beta} \mid \beta \in(\lambda, \zeta)\right\rangle$ is (strictly) increasing and $M\left[G_{0}\right]\left[H_{0}\right]\left[H_{1}\right]\left[H_{2}\right]$ is $\lambda$-closed with respect to $\bar{V}\left[G_{0}\right]\left[G_{1}\right]$, this definition is valid. Assuming now $r_{\zeta}$ has been defined and we wish to define $r_{\zeta+1}$, let $\left\langle\mathcal{B}_{\beta} \mid \beta<\eta \leq \lambda\right\rangle$ be the subsequence of $\left\langle\mathcal{A}_{\beta} \mid \beta \leq \zeta+1\right\rangle$ containing each antichain $\mathcal{A}$ such that $\mathcal{A} \subseteq \operatorname{Add}(j(\lambda), j(\zeta+1))$. Since $q_{\zeta}, r_{\zeta} \in \operatorname{Add}(j(\lambda), j(\zeta)), q_{\zeta+1} \in \operatorname{Add}(j(\lambda), j(\zeta+1))$, and $j(\zeta)<j(\zeta+1)$, the condition $r_{\zeta+1}^{\prime}=r_{\zeta} \cup q_{\zeta+1}$ is well-defined, since by our earlier observations, any new elements of $\operatorname{dom}\left(q_{\zeta+1}\right)$ won't be present in either $\operatorname{dom}\left(q_{\zeta}\right)$ or $\operatorname{dom}\left(r_{\zeta}\right)$. We can thus, using the fact $M\left[G_{0}\right]\left[H_{0}\right]\left[H_{1}\right]\left[H_{2}\right]$ is $\lambda$-closed with respect to $\bar{V}\left[G_{0}\right]\left[G_{1}\right]$, define by induction an increasing sequence $\left\langle s_{\beta} \mid \beta<\eta\right\rangle$ such that $s_{0} \geq r_{\zeta+1}^{\prime}, s_{\rho}=\bigcup_{\beta<\rho} s_{\beta}$ if $\rho$ is a limit ordinal, and $s_{\beta+1} \geq s_{\beta}$ is such that $s_{\beta+1}$ extends some element of $\mathcal{B}_{\beta}$. The just mentioned closure fact implies $r_{\zeta+1}=\bigcup_{\beta<\eta} s_{\beta}$ is a well-defined condition.

In order to show that $H_{3}$ is $M\left[G_{0}\right]\left[H_{0}\right]\left[H_{1}\right]\left[H_{2}\right]$-generic over $\operatorname{Add}(j(\lambda)$, $\left.j\left(\lambda^{+}\right)\right)$, we must show that $\forall \mathcal{A} \in\left\langle\mathcal{A}_{\zeta} \mid \zeta \in\left(\lambda, \lambda^{+}\right)\right\rangle \exists \beta \in\left(\lambda, \lambda^{+}\right) \exists r \in$ $\mathcal{A}\left[r_{\beta} \geq r\right]$. To do this, we first note that $\langle j(\zeta)| \zeta\left\langle\lambda^{+}\right\rangle$is unbounded in $j\left(\lambda^{+}\right)$. To see this, if $\beta<j\left(\lambda^{+}\right)$is an ordinal, then for some $f: \lambda \rightarrow M$ representing $\beta$, we can assume that for $\rho<\lambda, f(\rho)<\lambda^{+}$. Thus, by the regularity of $\lambda^{+}$in $\bar{V}, \beta_{0}=\bigcup_{\rho<\lambda} f(\rho)<\lambda^{+}$, and $j\left(\beta_{0}\right)>\beta$. This means by our earlier remarks that if $\mathcal{A} \in\left\langle\mathcal{A}_{\zeta} \mid \zeta<\lambda^{+}\right\rangle, \mathcal{A}=\mathcal{A}_{\rho}$, then we can let $\beta \in\left(\lambda, \lambda^{+}\right)$be such that $\mathcal{A} \subseteq \operatorname{Add}(j(\lambda), j(\beta))$. By construction, for $\eta>\max (\beta, \rho)$, there is some $r \in \mathcal{A}$ such that $r_{\eta} \geq r$. And, as any $p \in \operatorname{Add}\left(\lambda, \lambda^{+}\right)$is such that for some $\zeta \in\left(\lambda, \lambda^{+}\right), p=p\left\lceil\zeta, H_{3}\right.$ is such that if $p \in G_{1}, j(p) \in H_{3}$. Thus, working in $\bar{V}\left[G_{0}\right]\left[G_{1}\right]$, we have shown that $j$ lifts to $j: \bar{V}\left[G_{0}\right]\left[G_{1}\right] \rightarrow M\left[G_{0}\right]\left[H_{0}\right]\left[H_{1}\right]\left[H_{2}\right]\left[H_{3}\right]$, i.e., $\bar{V}\left[G_{0}\right]\left[G_{1}\right] \vDash " \lambda$ is measurable".

The proof of Theorem 5 will be finished once we have shown that $\bar{V}\left[G_{0}\right]\left[G_{1}\right] \vDash$ "No cardinal in the interval $(\delta, \lambda)$ is measurable". To see that this is the case, write $\mathbb{P}^{2}=\operatorname{Add}(\delta, 1) * \dot{\mathbb{R}}$. Since this definition shows that $\mathbb{P}^{2}$ admits a gap at $\delta$, by Theorem 4 , any cardinal in the interval $(\delta, \lambda)$ which is measurable in $\bar{V}\left[G_{0}\right]\left[G_{1}\right]$ had to have been measurable in $\bar{V}\left[G_{0}\right]$. However, since $\bar{V}\left[G_{0}\right] \vDash$ " $\lambda$ is the least measurable cardinal greater than $\delta$ ", $\bar{V}\left[G_{0}\right]\left[G_{1}\right] \vDash$ " $\lambda$ is the least measurable cardinal greater than $\delta$ " as well. This completes the proof of Theorem 5 . 
With the proof of Theorem 5 having been established, we can now prove Theorem 1. We follow the proofs of [2, Theorem 2] and [6, Theorem 1]. Suppose that $\kappa$ is indestructibly supercompact and there is a measurable cardinal $\lambda>\kappa$. We show that for any good ordinal $\alpha, A_{\alpha}=\{\delta<\kappa \mid \delta$ is measurable, $\delta$ is not a limit of measurable cardinals, and $\operatorname{LP}(\alpha)$ holds for $\delta$ \} is unbounded in $\kappa$. Let $\eta>\kappa$ be the least measurable cardinal. Force with $\mathbb{P}(\kappa, \eta, \alpha)$. After this forcing, which is $\kappa$-directed closed, $\operatorname{LP}(\alpha)$ holds for $\eta$, and $\eta$ remains the least measurable cardinal above $\kappa$. In particular, after the forcing, $\eta$ is a measurable cardinal which is not a limit of measurable cardinals at which $\operatorname{LP}(\alpha)$ holds. Since $\kappa$ is indestructibly supercompact, by reflection, $A_{\alpha}=\{\delta<\kappa \mid \delta$ is measurable, $\delta$ is not a limit of measurable cardinals, and $\operatorname{LP}(\alpha)$ holds for $\delta\}$ is unbounded in $\kappa$ after the forcing has been performed. Once more, we infer by the fact $\mathbb{P}(\kappa, \eta, \alpha)$ is $\kappa$-directed closed that $A_{\alpha}$ is unbounded in $\kappa$ in the ground model. This completes the proof of Theorem 1 .

Having finished the proof of Theorem 1, we turn now to the proof of Theorem 2.

Proof. Suppose $V \vDash$ "ZFC $+\kappa$ is supercompact + No cardinal $\zeta>\kappa$ is measurable". Let $\alpha$ be a fixed but arbitrary good ordinal. Take $\left\langle\delta_{j} \mid j<\kappa\right\rangle$ to be the continuous, increasing enumeration of $\{\omega\} \cup\{\delta<\kappa \mid \delta$ is either a measurable cardinal or a limit of measurable cardinals $\}$. For any measurable cardinal $\delta, \delta=\delta_{j}$, let $\theta_{\delta}$ be the least cardinal $\theta \in\left(\delta_{j}, \delta_{j+1}\right)$ such that $\delta$ is not $\theta$ supercompact in $V$, or $\delta$ if $\delta$ is $\delta_{j+1}$ supercompact in $V$. We define now a length $\kappa$ reverse Easton iteration $\mathbb{P}=\left\langle\left\langle\mathbb{P}_{\delta}, \dot{\mathbb{Q}}_{\delta}\right\rangle \mid \delta<\kappa\right\rangle$ by four cases as follows, taking as an inductive hypothesis that if $\delta=\delta_{j}$ is a measurable cardinal, then $\Vdash_{\mathbb{P}_{\delta}}$ " $\delta_{j+1}$ is the least measurable cardinal greater than $\delta$ ":

(1) $\mathbb{P}_{0}=\operatorname{Add}(\omega, 1)=\operatorname{Add}\left(\delta_{0}, 1\right)$.

(2) If $\delta=\omega$ or $\delta$ is in $V$ either a non-measurable limit of measurable cardinals or a measurable cardinal which is not a limit of measurable cardinals, let $j$ be such that $\delta=\delta_{j}$. Then $\mathbb{P}_{\delta+1}=\mathbb{P}_{\delta} * \dot{\mathbb{Q}}_{\delta}$, where $\dot{\mathbb{Q}}_{\delta}$ is a term for the partial ordering $\mathbb{P}\left(\delta_{j}^{++}, \delta_{j+1}, \alpha\right)$ of Theorem 5 .

(3) If $\delta$ is in $V$ a measurable limit of measurable cardinals with $\delta=\delta_{j}$, then $\mathbb{P}_{\delta+1}=\mathbb{P}_{\delta} * \dot{\mathbb{Q}} * \dot{\mathbb{P}}\left(\dot{\eta}, \delta_{j+1}, \alpha\right)=\mathbb{P}_{\delta} * \dot{\mathbb{Q}}_{\delta}$. Here, $\Vdash_{\mathbb{P}_{\delta}}$ "बi் is the 
lottery sum of all $\delta$-directed closed partial orderings having rank less than $\delta_{j+1}$ ", and $\Vdash_{\mathbb{P}_{\delta} * \dot{\mathbb{Q}}}$ " $\dot{\eta}$ is the least inaccessible cardinal greater than $\max \left(\theta_{\delta},|\mathrm{TC}(\dot{\mathbb{R}})|\right)$, where $\dot{\mathbb{R}}$ is the partial ordering selected in the stage $\delta$ lottery".

(4) If neither Cases $1-3$ holds, then $\mathbb{P}_{\delta+1}=\mathbb{P}_{\delta} * \dot{\mathbb{Q}}_{\delta}$, where $\dot{\mathbb{Q}}_{\delta}$ is a term for trivial forcing $\{\emptyset\}$.

By induction, it follows that for any $j<\kappa, \mathbb{P}_{\delta_{j}}$ is forcing equivalent to a partial ordering having size at most $2^{\delta_{j}}<\delta_{j+1}$. From this, the Lévy-Solovay results [19] show that the inductive hypothesis holds and $\mathbb{P}$ is well-defined.

Lemma 2.1. $V^{\mathbb{P}} \vDash " \kappa$ is indestructibly supercompact".

Proof. We slightly modify the proofs of [2, Lemma 2.1], [6, Lemma 2.1], [7, Lemma 3.1], and [1, Lemma 2.6], quoting verbatim when appropriate. Let $\mathbb{Q} \in V^{\mathbb{P}}$ be such that $V^{\mathbb{P}} \vDash$ " $\mathbb{Q}$ is $\kappa$-directed closed". Take $\dot{\mathbb{Q}}$ as a term for $\mathbb{Q}$ such that $\vdash_{\mathbb{P}}$ "⿺辶冋 is $\kappa$-directed closed". Suppose $\lambda \geq|\mathrm{TC}(\dot{\mathbb{Q}})|$ is an arbitrary cardinal, and let $\gamma=2^{\mid[\lambda]^{<\kappa \mid}}$. Take $j: V \rightarrow M$ as an elementary embedding witnessing the $\gamma$ supercompactness of $\kappa$ generated by a supercompact ultrafilter over $P_{\kappa}(\gamma)$ such that $M \vDash " \kappa$ is not $\gamma$ supercompact". Since $V \vDash$ "No cardinal $\zeta>\kappa$ is measurable" and $M^{\gamma} \subseteq M$, the definition of $\mathbb{P}$ implies that in $M$, above the appropriate condition, $j(\mathbb{P} * \dot{\mathbb{Q}})$ is forcing equivalent to $\mathbb{P} * \dot{\mathbb{Q}} * \dot{\mathbb{R}} * j(\dot{\mathbb{Q}})$, where the first stage at which $\dot{\mathbb{R}}$ is forced to do nontrivial forcing is well above $\gamma$. Laver's original argument from [17] now applies and shows that $V^{\mathbb{P} * \dot{\mathbb{Q}}} \vDash$ " $\kappa$ is $\lambda$ supercompact". (Simply let $G_{0} * G_{1} * G_{2}$ be $V$-generic over $\mathbb{P} * \dot{\mathbb{Q}} * \dot{\mathbb{R}}$, lift $j$ in $V\left[G_{0}\right]\left[G_{1}\right]\left[G_{2}\right]$ to $j: V\left[G_{0}\right] \rightarrow M\left[G_{0}\right]\left[G_{1}\right]\left[G_{2}\right]$, take a master condition $p$ for $j^{\prime \prime} G_{1}$ and a $V\left[G_{0}\right]\left[G_{1}\right]\left[G_{2}\right]$-generic object $G_{3}$ over $j(\mathbb{Q})$ containing $p$, lift $j$ again in $V\left[G_{0}\right]\left[G_{1}\right]\left[G_{2}\right]\left[G_{3}\right]$ to $j: V\left[G_{0}\right]\left[G_{1}\right] \rightarrow M\left[G_{0}\right]\left[G_{1}\right]\left[G_{2}\right]\left[G_{3}\right]$, and show by the $\gamma^{+}$-directed closure of $\mathbb{R} * j(\dot{\mathbb{Q}})$ that the supercompactness measure over $\left(P_{\kappa}(\lambda)\right)^{V\left[G_{0}\right]\left[G_{1}\right]}$ generated by $j$ is actually a member of $V\left[G_{0}\right]\left[G_{1}\right]$.) As $\lambda$ and $\mathbb{Q}$ were arbitrary, this completes the proof of Lemma 2.1.

Lemma 2.2. $V^{\mathbb{P}} \vDash$ "If $\delta<\kappa$ is a measurable cardinal which is not a limit of measurable cardinals, then LP $(\alpha)$ holds". 
Proof. Let $\gamma<\kappa$ be such that $V \vDash " \delta=\delta_{\gamma}$ is a measurable cardinal which is not a limit of measurable cardinals". By the definition of the $\delta_{j}$ 's, it must be the case that $\gamma$ is a successor ordinal. Let $\gamma=\beta+1, \sigma=\delta_{\beta}$, and write $\mathbb{P}=\mathbb{P}_{\sigma+1} * \dot{\mathbb{P}}^{\sigma+1}$. By the definition of $\mathbb{P}$ and Theorem 4, it must also be true that $V^{\mathbb{P}_{\sigma+1}} \vDash " \delta_{\beta+1}$ is the least measurable cardinal greater than $\delta_{\beta}+\operatorname{LP}(\alpha)$ holds for $\delta_{\beta+1}$ ". ${ }^{3}$ Since $\Vdash_{\mathbb{P}_{\sigma+1}}$ " $\mathbb{P}^{\sigma+1}$ is $\delta_{\beta+1}^{++}$-directed closed", $V^{\mathbb{P}_{\sigma+1} * \dot{\mathbb{P}}^{\sigma+1}}=V^{\mathbb{P}} \vDash " \delta_{\beta+1}$ is the least measurable cardinal greater than $\delta_{\beta}$ $+\operatorname{LP}(\alpha)$ holds for $\delta_{\beta+1}$ " as well. The proof of Lemma 2.2 will therefore be complete once we have shown that in $V^{\mathbb{P}}$, any measurable cardinal $\delta<\kappa$ which is not a limit of measurable cardinals is such that $\delta=\delta_{\beta+1}$ for some $\beta<\kappa$.

To see this, assume to the contrary that $\delta \neq \delta_{\beta+1}$ for any $\beta<\kappa$. Write $\mathbb{P}=\mathbb{P}_{0} * \dot{\mathbb{Q}}$, where $\mathbb{P}_{0}=\operatorname{Add}(\omega, 1)$ and $\Vdash_{\mathbb{P}_{0}}$ "⿺辶் is $\aleph_{2}$-directed closed". Since $\mathbb{P}$ admits a gap at $\omega$, by Theorem 4 , any cardinal measurable in $V^{\mathbb{P}}$ had to have been measurable in $V$. This means that $\delta=\delta_{\lambda}$ for some limit ordinal $\lambda<\kappa$, i.e., in $V, \delta$ is a measurable limit of measurable cardinals. In particular, in $V, \delta$ is a limit of measurable cardinals which are not themselves limits of measurable cardinals. It consequently follows that in $V, \delta$ is a limit of measurable cardinals which have the form $\delta_{\beta+1}$ for some $\beta<\kappa$. However, the arguments of the preceding paragraph show that any such measurable cardinal remains measurable in $V^{\mathbb{P}}$. From this, we immediately infer that in $V^{\mathbb{P}}, \delta$ is a measurable limit of measurable cardinals. This contradiction completes the proof of Lemma 2.2.

Since $\mathbb{P}$ may be defined so that $|\mathbb{P}|=\kappa$, by the results of [19], $V^{\mathbb{P}} \vDash$ "No cardinal $\zeta>\kappa$ is measurable". This fact, together with Lemmas 2.1 and 2.2 , complete the proof of Theorem 2 .

\footnotetext{
${ }^{3}$ If we are in Case 2 of the definition of $\mathbb{P}$ at stage $\sigma+1$, then this follows by the results of [19], since $\mathbb{P}_{\delta_{\beta}}$ is forcing equivalent to a partial ordering having size at most $2^{\delta_{\beta}}<\delta_{\beta+1}$. If we are in Case 3 of the definition of $\mathbb{P}$ at stage $\sigma+1$, with $\mathbb{P}_{\sigma+1}=\mathbb{P}_{\sigma} * \dot{\mathbb{Q}} * \dot{\mathbb{P}}\left(\dot{\eta}, \delta_{\beta+1}, \alpha\right)$, then because forcing with $\mathbb{Q}$ is forcing equivalent to forcing with a partial ordering having size less than $\delta_{\beta+1}$, an application of the results of [19] shows that $\Vdash_{\mathbb{P}_{\sigma * \dot{\mathbb{Q}}}}$ " $\delta_{\beta+1}$ is measurable". Because $\mathbb{P}_{\sigma} * \dot{\mathbb{Q}}=\mathbb{P}_{0} * \dot{\mathbb{R}}$, where $\mathbb{P}_{0}=\operatorname{Add}(\omega, 1)$ and $\vdash_{\mathbb{P}_{0}}$ " $\mathbb{R}$ is $\aleph_{2}$-directed closed", $\mathbb{P}_{\sigma} * \dot{\mathbb{Q}}$ admits a gap at $\omega$. Therefore, by Theorem 4 , any cardinal measurable in $V^{\mathbb{P}_{\sigma} * \mathbb{Q}}$ had to have been measurable in $V$. This means that $\vdash_{\mathbb{P}_{\sigma * \mathbb{Q}}}$ " $\delta_{\beta+1}$ is the least measurable cardinal greater than $\delta_{\beta}$ ". This fact is then preserved after forcing with $\mathbb{P}\left(\eta, \delta_{\beta+1}, \alpha\right)$.
} 
Having finished the proof of Theorem 2, we turn now to the proof of Theorem 3.

Proof. Suppose $V \vDash$ "ZFC $+\kappa$ is indestructibly supercompact + No cardinal $\zeta>\kappa$ is measurable". Without loss of generality, by first forcing GCH if necessary and then forcing with the (possibly proper class) reverse Easton iteration which is trivial except at inaccessible stages $\delta$, where the partial ordering used is $\operatorname{Add}\left(\delta, \delta^{++}\right)$, we may assume in addition that $V \vDash " 2^{\delta}=\delta^{++}$ for every inaccessible cardinal $\delta$ ".

As in the proof of Theorem 2, let $\left\langle\delta_{j} \mid j<\kappa\right\rangle$ be the continuous, increasing enumeration of $\{\omega\} \cup\{\delta<\kappa \mid \delta$ is either a measurable cardinal or a limit of measurable cardinals $\}$. We define now a length $\kappa$ reverse Easton iteration $\mathbb{P}=\left\langle\left\langle\mathbb{P}_{\delta}, \dot{\mathbb{Q}}_{\delta}\right\rangle \mid \delta<\kappa\right\rangle$ by three cases as follows:

(1) $\mathbb{P}_{0}=\operatorname{Add}(\omega, 1)=\operatorname{Add}\left(\delta_{0}, 1\right)$.

(2) If $\delta$ is in $V$ a measurable limit of measurable cardinals with $\delta=\delta_{j}$, then $\mathbb{P}_{\delta+1}=\mathbb{P}_{\delta} * \dot{\mathbb{Q}}_{\delta}$, where $\Vdash_{\mathbb{P}_{\delta}}$ " $\dot{\mathbb{Q}}_{\delta}$ is the lottery sum of all $\delta$ directed closed partial orderings having rank less than $\delta_{j+1}$ ".

(3) If neither Cases 1 nor 2 holds, then $\mathbb{P}_{\delta+1}=\mathbb{P}_{\delta} * \dot{\mathbb{Q}}_{\delta}$, where $\dot{\mathbb{Q}}_{\delta}$ is a term for trivial forcing $\{\emptyset\}$.

The same reasoning as given for Theorem 2 allows us to infer that $V^{\mathbb{P}} \vDash$ " $\kappa$ is indestructibly supercompact + No cardinal $\zeta>\kappa$ is measurable". The proof of Theorem 3 will therefore be completed by the following lemma.

Lemma 2.3. $V^{\mathbb{P}} \vDash$ "If $\delta<\kappa$ is a measurable cardinal which is not a limit of measurable cardinals, then $2^{\delta}=\delta^{++"}$.

Proof. We argue in analogy to the proof of Lemma 2.2. Let $\gamma<\kappa$ be such that $V \vDash " \delta=\delta_{\gamma}$ is a measurable cardinal which is not a limit of measurable cardinals". As before, by the definition of the $\delta_{j}$ 's, it must be the case that $\gamma$ is a successor ordinal. Let $\gamma=\beta+1, \sigma=\delta_{\beta}$, and write $\mathbb{P}=\mathbb{P}_{\sigma+1} * \dot{\mathbb{P}}^{\sigma+1}$. By the definition of $\mathbb{P}$, it inductively follows that $\mathbb{P}_{\sigma+1}$ is forcing equivalent to a partial ordering having size less than $\delta_{\beta+1}$. Since $\Vdash_{\mathbb{P}_{\sigma+1}}$ " $\dot{\mathbb{P}}^{\sigma+1}$ is (at least) $\delta^{+3}$-directed closed", in both $V^{\mathbb{P}_{\sigma+1}}$ and $V^{\mathbb{P}_{\sigma+1} * \dot{\mathbb{P}}^{\sigma+1}}=V^{\mathbb{P}}, \delta$ is a measurable cardinal which is not a limit of measurable cardinals and $2^{\delta}=\delta^{++}$. The same proof as given in Lemma 2.2 now shows that if $V^{\mathbb{P}} \vDash " \delta$ is a measurable 
cardinal which is not a limit of measurable cardinals", then $\delta=\delta_{\rho+1}$ for some $\rho<\kappa$. This completes the proof of both Lemma 2.3 and Theorem 3 .

In conclusion to this paper, we note that Theorems $1-3$ remain valid if the definition of good ordinal is changed to allow $\delta^{+\alpha}$ to be a regular cardinal above the least inacessible cardinal greater than $\delta$. The definition used in this paper was chosen as a matter of convenience and ease of presentation. In addition, we observe that results analogous to Theorems $1-3$ hold if $\kappa$ is either an indestructible strong cardinal in Gitik and Shelah's sense of [10] or an indestructible strongly unfoldable cardinal in Johnstone's sense of $[15,16]$. (See $[15,16]$ for the definition of strongly unfoldable cardinal.) Readers may work out the details for themselves.

\section{REFERENCES}

[1] A. Apter, Indestructibility and destructible measurable cardinals, Arch. Math. Logic, 55 (2016), 3-18.

[2] A. Apter, Indestructibility and level by level equivalence and inequivalence, Math. Log. Q., 53 (2007), 78-85.

[3] A. Apter, Indestructibility and measurable cardinals with few and many measures, Arch. Math. Logic, 47 (2008), 101-110.

[4] A. Apter, Indestructibility and stationary reflection, Math. Log. Q., 55 (2009), 228236.

[5] A. Apter, Indestructibility, HOD, and the ground axiom, Math. Log. Q., 57 (2011), 261-265.

[6] A. Apter, Indestructibility, instances of strong compactness, and level by level inequivalence, Arch. Math. Logic, 49 (2010), 725-741.

[7] A. Apter, Indestructibilty, measurability, and degrees of supercompactness, Math. Log. Q., 58 (2012), 75-82.

[8] A. Apter and J. D. Hamkins, Indestructibility and the level-by-level agreement between strong compactness and supercompactness, J. Symb. Log., 67 (2002), 820-840.

[9] A. Apter and S. Shelah, On the strong equality between supercompactness and strong compactness, Trans. Am. Math. Soc., 349 (1997), 103-128.

[10] M. Gitik and S. Shelah, On certain indestructibility of strong cardinals and a question of Hajnal, Arch. Math. Log., 28 (1989), 35-42.

[11] J. D. Hamkins, Gap forcing, Israel J. Math., 125 (2001), 237-252.

[12] J. D. Hamkins, Gap forcing: generalizing the Lévy-Solovay theorem, Bull. Symb. Log., 5 (1999), 264-272.

[13] J. D. Hamkins, The lottery preparation, Ann. Pure Appl. Log., 101 (2000), 103-146.

[14] T. Jech, Set Theory. The Third Millennium Edition, Revised and Expanded, SpringerVerlag, Berlin and New York, 2003. 
[15] T. Johnstone, Strongly Unfoldable Cardinals Made Indestructible, Doctoral Dissertation, the CUNY Graduate Center, 2007.

[16] T. Johnstone, Strongly unfoldable cardinals made indestructible, J. Symb. Log., 73 (2008), 1215-1248.

[17] R. Laver, Making the supercompactness of $\kappa$ indestructible under $\kappa$-directed closed forcing, Israel J. Math., 29 (1978), 385-388.

[18] J. -P. Levinski, Filters and large cardinals, Ann. Pure Appl. Log., 72 (1995), 177-212.

[19] A. Lévy and R. Solovay, Measurable cardinals and the continuum hypothesis, Israel J. Math., 5 (1967), 234-248.

[20] M. Magidor, On the existence of nonregular ultrafilters and the cardinality of ultrapowers, Trans. Am. Math. Soc., 249 (1979), 97-111.

(Received: May 29, 2015)

(Revised: September 12, 2015)
Department of Mathematics

Baruch College of CUNY

New York, New York 10010, U.S.A.

and

The CUNY Graduate Center, Mathematics 365 Fifth Avenue

New York, New York 10016 U.S.A.

http://faculty.baruch.cuny.edu/aapter

awapter@alum.mit.edu 